\title{
Reprogrammed CRISPR-Cas13a targeting the HPV16/18 E6 gene inhibits proliferation and induces apoptosis in E6-transformed keratinocytes
}

\author{
CHUNJING LI $^{1 *}$, LIWEN GUO ${ }^{2 *}$, GUOQING LIU ${ }^{1}$, MINGJUAN GUO ${ }^{1}$, HUILING WEI $^{1}$, \\ QIQIONG YANG ${ }^{1}$, JIANFENG WANG ${ }^{1}$ and HUIHUA CHEN ${ }^{2}$ \\ ${ }^{1}$ Department of Urology, Foshan Maternal and Child Health Hospital, Southern Medical University, \\ Foshan, Guangdong 528000; ${ }^{2}$ Department of Gynecology, Ningde Municipal Hospital, \\ Fujian Medical University, Ningde, Fujian 352100, P.R. China
}

Received August 30, 2019; Accepted March 4, 2020

DOI: $10.3892 /$ etm.2020.8631

\begin{abstract}
The long-lasting infection of high-risk type (type 16 or type 18) human papillomavirus (HPV) is the main cause of gynecological and urinary malignancies. Given the high mortality rate after surgery, the development of a new molecular therapy would be of value to clinicians. The aim of the present study was to achieve targeted inactivation of the viral E6 gene in keratinocytes using the reprogrammed CRISPR-Cas13a system. To accomplish this, a reprogrammed CRISPR-Cas13a system, targeting both the HPV16/18 E6 genes was constructed using a guide RNA expressing vector. The expression levels of E6 protein were measured using western blot analysis after transfection of the vector into E6-transformed keratin oocytes. Cell proliferation was analyzed using CCK-8 assays and cell apoptosis was evaluated using Hoechst 33258 staining and ELISAs examining caspase-3 levels. The results indicated that both the HPV16/18 E6 genes can be inactivated using the CRISPR-Cas13a system. Furthermore, silencing E6 inhibited cell proliferation $(14 \pm 1.8 \%$ on average) and induced apoptosis ( $80.2 \pm 3.2 \%$ on average) in E6-transformed keratinocytes but not in normal keratinocytes. In conclusion, results of the present study demonstrate that the
\end{abstract}

Correspondence to: Dr Jianfeng Wang, Department of Urology, Foshan Maternal and Child Health Hospital, Southern Medical University, 11 Renmin West Road, Foshan, Guangdong 528000, P.R. China

E-mail: jianfengwang1977@aliyun.com

Dr Huihua Chen, Department of Gynecology, Ningde Municipal Hospital, Fujian Medical University, 7 Jiaocheng North Road, Ningde, Fujian 352100, P.R. China

E-mail: 183757610@qq.com

*Contributed equally

Key words: CRISPR-Cas13a, human papilloma virus 16/18 E6 gene, keratinocytes, proliferation, apoptosis reprogrammed CRISPR-Cas13a system has the potential for inactivating HPV E6 gene functions.

\section{Introduction}

Human papillomaviruses (HPVs) are important sexually transmitted human pathogens (1). The most common high-risk HPV types, 16 and 18, are frequently detected in gynecological and urological cancers (2). The low risk HPV types, 6 and 11, are associated with anogenital warts and laryngeal papillomatosis (3). HPV-related lesions require continued production of the oncogenic E6 protein, which has targets in both the nucleus and cytoplasm, and promotes cell proliferation in human keratinocytes (4). Small molecule inhibitors and small interfering RNAs (siRNAs) targeting the E6 oncogene can be used to improve the therapeutic effects of current therapies for treating HPV-related gynecological and urological diseases (5).

RNA-guided, RNA degradation is a natural phenomenon mediated by the bacterial type II CRISPR/CRISPR-associated protein (Cas)13a system (6). This system can be reprogrammed to induce mRNA and long non-coding RNA knockdown at specific sites (7). The potential of CRISPR-Cas13a to treat or diagnose human genetic diseases has yet to be demonstrated. Although it has been revealed that the E6 gene can be inactivated by CRISPR-Cas9 $(8,9)$, to the best of our knowledge, there are no reports regarding the study of a designed CRISPR-Cas13a targeted against the E6 gene of HPV 16/18, the pathogen behind gynecological and urological cancers. Both CRISPR-Cas9 and siRNA may cause significant off-target effects (7), while the targeting specificity of CRISPR-Cas13a is considered to be excellent. Therefore, CRISPR-Cas13a may be a new and useful molecular tool for targeting the HPV E6 gene.

To test the potential application value of CRISPR-Cas13a, the present study constructed a CRISPR-Cas13a system that targets the sequence of the HPV16/18 E6 gene. The aim of the present study was to fully investigate the feasibility of using CRISPR-Cas13a to degrade E6 mRNA and resist the pathogenic effects of HPVs. The study on the silencing effect of CRISPR-Cas13a on the pathogenic genes of HPV may bring new means for the treatment of HPV-related infectious diseases. 


\section{Materials and methods}

Cell lines and cell culture. Human keratinocytes were isolated from the neonatal foreskin by two-step digestion using trypsin and dispase II and grown in RPMI-1640 media supplemented with $10 \%$ FBS (both from Invitrogen; Thermo Fisher Scientific, Inc.) at $37^{\circ} \mathrm{C}$ in an atmosphere of $5 \% \mathrm{CO}_{2}$. Informed patient consent was obtained from the parents of the newborn and the patient protocols were approved by the institutional review board of the Foshan Maternal and Child Health Hospital (approval no. 201914258).

Construction of plasmids. To construct a reprogrammed CRISPR/Cas13a system that targets the entire E6 protein of HPV 16/18, two different single guide RNA (sgRNA) sequences (Fig. 1B) were designed using the CHOPCHOP v3 online tool (https://chopchop.cbu.uib.no) and inserted into the sgRNA expression cassettes of the pCRISPR-CG01 vector (cat. no. PVT10921; Guangzhou FulenGen, Co., Ltd.) containing the U6 promoter to drive the transcription of each sgRNA, as well as the cytomegalovirus (CMV) promoter to drive the expression of the Cas13a protein. The same vector, however, expressing sgRNAs that lack the complete complementary region, was also constructed and used as the negative control.

To construct the plasmids pcDNA3.1-HPV16-E6 and pcDNA3.1-HPV18-E6, the coding sequences for HPV16 E6 and HPV18 E6, were chemically synthesized and inserted into pcDNA3.1 (+), digested with HindIII and EcoRI, respectively.

Cell transfections. For stable transfection experiments, human keratinocytes stably expressing the HPV16 E6 or HPV18 E6 gene were obtained by selecting cells using the G418 drug after transfection with the related plasmids. For transient transfection experiments, the cells $\left(5 \times 10^{5}\right)$ were cultured in the 12 -well plates until they reached $70 \%$ confluency. They were then transfected with $1.25 \mu \mathrm{g}$ Cas13a- and sgRNAs-expressing plasmids using Lipofectamine ${ }^{\circledR} 2000$ (Invitrogen; Thermo Fisher Scientific, Inc.) according to the supplier's protocols. Cells were used for further experimentation after 24-72 $\mathrm{h}$ following transfection.

Western blot analysis. Cells were washed in ice-cold PBS and lysed in modified RIPA buffer (Amresco, LLC). The protein concentration was calculated using the bicinchoninic acid protein assays. A total of $40 \mu \mathrm{g}$ protein extract per lane were separated by $10 \%$ SDS-polyacrylamide gels and transferred onto PVDF membranes (EMD Millipore). Membranes were blocked overnight at $4^{\circ} \mathrm{C}$ in $5 \%$ non-fat milk in TBS and incubated overnight with the mouse anti-HPV16 E6 + HPV18 E6 antibodies (cat. no. ab70; 1:10,000; Abcam) at $4^{\circ} \mathrm{C}$. Subsequently, the membranes were incubated with horseradish peroxidase-conjugated anti-mouse secondary antibodies at 1:10,000 dilution (cat. no. NA931; GE Healthcare Life Sciences) and the immunoblots were developed using Super-Signal chemiluminescence reagents (Pierce; Thermo Fisher Scientific, Inc.).

Cell proliferation assay. Cell proliferation was examined using CCK-8 assays according to the manufacturer's protocol (Beyotime Institute of Biotechnology). After 24, 48 or $72 \mathrm{~h}$ post-transfection, $10 \mu \mathrm{lCCK}-8$ solution was added to each well of the 96-well plates and the cells were maintained for $4 \mathrm{~h}$ at $37^{\circ} \mathrm{C}$.
Absorbance values were determined at a wavelength of $450 \mathrm{~nm}$ using an ELISA microplate reader (Bio-Rad Laboratories, Inc.). The assay was repeated at least three times independently.

Cell apoptosis assay. Morphological assessment of apoptotic cells was performed using a Hoechst-33258 staining kit (Beyotime Institute of Biotechnology) according to the supplier's protocols. Briefly, the cells were fixed in $4 \%$ paraformaldehyde for $10 \mathrm{~min}$ at room temperature, and were washed twice with PBS. The cells were then stained using $0.5 \mathrm{ml}$ Hoechst-33258 for $5 \mathrm{~min}$ at room temperature, and were subsequently observed using a fluorescence microscope (magnification, x100; Nikon Corporation) at $350 \mathrm{~nm}$. Fluorescence images were captured from six randomly chosen fields per well and each experiment was repeated three times).

Cell apoptosis was examined by analyzing the activity of Caspase-3 using the enzyme-linked immunosorbent assay kit (R\&D Systems, Inc.) according to the manufacturer's instructions. The optical density (OD) values were measured using an ELISA microplate reader (Bio-Rad Laboratories, Inc.).

Statistical analyses. Data are expressed as the mean \pm SD. Statistical significance was determined using Student's t-tests or ANOVAs with post hoc Tukey's tests. $\mathrm{P}<0.05$ was considered to indicate a statistically significant difference. All the related statistical tests were performed using SPSS version 17.0 software (SPSS, Inc.).

\section{Results}

Design and construction of the reprogrammed CRISPR-Cas 13 a system. To determine whether CRISPR-Cas13a could silence the HPV16/18 E6 gene, a single plasmid was constructed to express the Cas13a and guide RNAs that can be used to make cuts at designated sites (Fig. 1A). The targets of the sgRNAs were designed to select sequences of the E6 genes of HPV16/18 (Fig. 1B). A CRISPR-Cas13a system that expresses sgRNAs lacking the complementary regions was used as the negative control.

Promotion of cell growth by the HPV16/18 E6 gene. Plasmids encoding HPV16 E6 and HPV18 E6 (Fig. S1) were first constructed, following which protein overexpression was verified by western blotting. The potential alteration of cellular phenotypes after HPV16/18 E6 overexpression was examined by stable transfection of the corresponding plasmids. As shown in Fig. 2, consistent with results from a previous study (4), the E6 gene of HPV16 or HPV18 significantly promoted cell growth $(\mathrm{P}<0.01)$, as indicated by $\mathrm{OD}$ values when compared with the OD values of normal keratinocytes transfected with the empty pcDNA3.1 (+) vector. The transformed keratinocytes were then used as test models for analyzing the effects of the reprogrammed CRISPR-Cas13a system on the HPV16/18 E6 genes in the subsequent experiments.

Inhibition of HPV16/18 E6 expression by the reprogrammed CRISPR-Cas13a. We then investigated whether the endogenous HPV-16/18 E6 genes can be inactivated in the transformed keratinocytes using the reprogrammed CRISPR-Cas13a system. Plasmids expressing the specific CRISPR-Cas13a or the negative control were transiently transfected into keratinocytes. The 
A

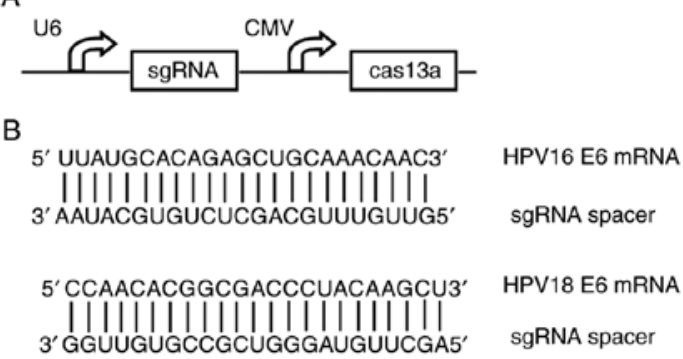

Figure 1. Design and construction of the reprogrammed CRISPR-Cas13a system. (A) Detailed description of the engineered vector for expressing the CRISPR-Cas13a system. (B) Diagram of the two sequences targeted by sgRNAs and the related PAM sequences. Cas, CRISPR-associated protein; HPV, human papillomavirus; sgRNA, single guide RNA; CMV, cytomegalovirus; PAM, protospacer adjacent motif.

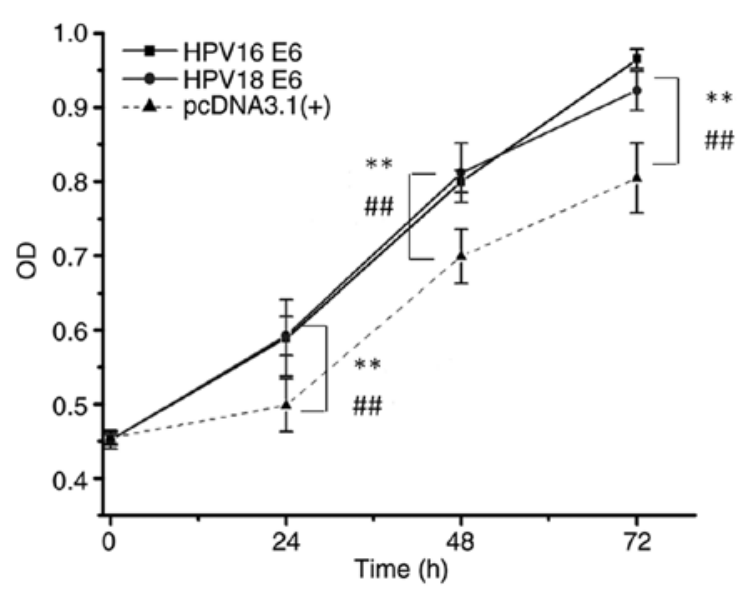

Figure 2. Overexpression of the HPV 16/18 E6 genes in keratinocytes. Compared to keratinocytes transfected with the empty pcDNA3.1 (+) vector, keratinocytes transformed with the HPV16 E6 gene or the HPV18 E6 gene showed an increased OD, representing an increased cell proliferation. ${ }^{* * *} \mathrm{P}<0.01$, pcDNA3.1 vs. HPV $16 ;{ }^{\# \#} \mathrm{P}<0.01$, pcDNA3.1 vs. HPV 18 . HPV, human papillomavirus; $\mathrm{OD}$, optical density.

expression levels of E6 proteins were then monitored at 48-h post-transfection using western blot analyses. Interestingly, expression of the single CRISPR-Cas13a system resulted in a marked loss of both the HPV16 E6 protein (Fig. 3A) and the HPV18 E6 protein (Fig. 3B). It was therefore concluded that the reprogrammed CRISPR-Cas13a system could effectively silence the expression of the HPV16/18 E6 genes.

Inhibition of proliferation of the E6-transformed keratinocytes by the reprogrammed CRISPR-Cas13a. E6 expression is known to be essential for HPV-transformed cell growth and survival (4). Therefore, whether the proliferation of E6-transformed keratinocytes can be affected by the reprogrammed CRISPR-Cas13a was examined.

The keratinocytes transformed with the HPV16/18 E6 genes were transiently transfected with the reprogrammed CRISPR-Cas13a or the negative control in 96-well plates. In CCK-8 assays, it was demonstrated that the proliferative ability of keratinocytes was decreased (by $14 \pm 1.8 \%$ on average; $\mathrm{P}<0.01)$ when cells were treated with the reprogrammed
A

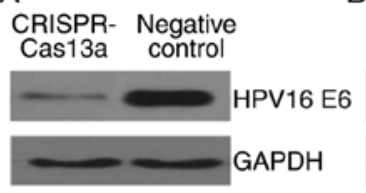

B

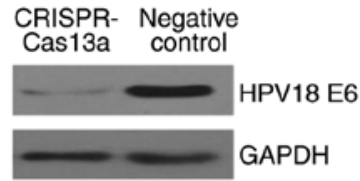

Figure 3. Detection of E6 protein expression levels in keratinocytes treated with the reprogrammed CRISPR-Cas13a system. Western-blot analysis was performed to detect the expression levels of (A) HPV16 E6 or (B) HPV18 E6 in keratinocytes transfected with either the reprogrammed CRISPR-Cas13a system or the negative control. Cas, CRISPR-associated protein; HPV, human papillomavirus.

CRISPR-Cas13a (Fig. 4A and B). Additionally, the same effects were not observed in the negative control keratinocytes $(\mathrm{P}>0.05)$ that were stably transfected with the empty pcDNA3.1 (+) vector (Fig. 4C), indicating that the reprogrammed CRISPR-Cas13a does not have off-target effects.

Induction of apoptosis of the E6-transformed keratinocytes by the reprogrammed CRISPR-Cas13a. Finally, whether the apoptosis of E6-transformed keratinocytes can be affected by the reprogrammed CRISPR-Cas13a was also examined. Forty-eight hours after transfection of the reprogrammed CRISPR-Cas13a or negative control, changes in the rate of apoptosis of the E6-transformed keratinocytes were determined using Hoechst-33258 staining and ELISAs. Cells transfected with the reprogrammed CRISPR-Cas13a exhibited strong blue fluorescence in the nucleolus after staining with Hoechst 33258, revealing the apoptotic characteristics of the cells (Fig. 5A). An increase in caspase 3 levels $(80.2 \pm 3.2 \%$ on average; $\mathrm{P}<0.05)$ was also observed in both the HPV16/18 E6-transformed keratinocytes transfected with the reprogrammed CRISPR-Cas13a (Fig. 5B). The reprogrammed CRISPR-Cas13a did not induce apoptosis $(\mathrm{P}>0.05)$ in keratinocytes stably transfected with the empty plasmid (Fig. 5A and B).

\section{Discussion}

The current treatments for gynecological and urological tumors usually include surgical removal, chemotherapy and radiotherapy (10). Although there are multiple approaches for treating these diseases, the rate of recurrence and fatality after successful clearance is still very high (10). Further improvements in treatment outcomes may evolve from combinations of conventional therapy with novel molecular agents (5). The expression of the HPV E6 protein is positively associated with uncontrolled proliferation of keratinocytes during the development of gynecological and urological tumors (5). With this in mind, the E6 gene is an ideal target for molecular agents targeting HPV 16/18. In previous studies, anti-sense oligodeoxynucleotides (11), ribozymes (12) and siRNA (13) have already been constructed to inhibit the expression levels of E6 mRNA/protein. However, those approaches have only achieved limited success due to the low accessibility of most sites on the HPV16/18 E6 mRNA (11). Therefore, treatments based on new molecular agents should be developed.

The recent advancements in CRISPR engineering have resulted in the development of more targeted and potentially 

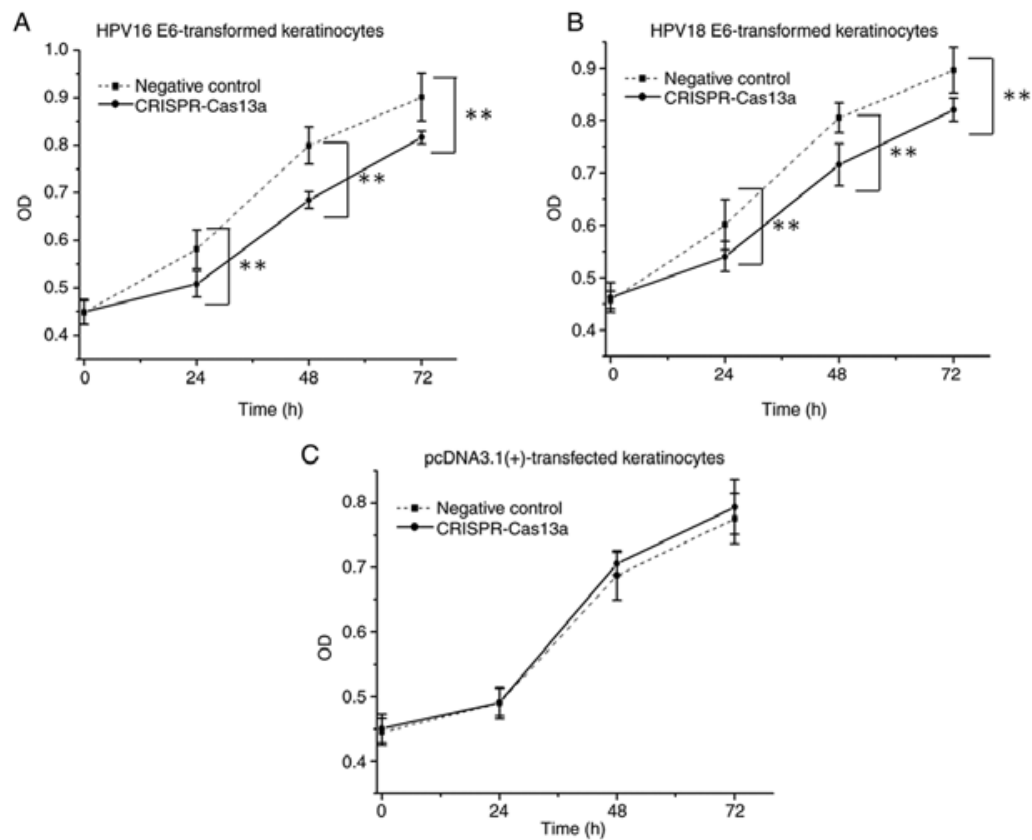

Figure 4. The CRISPR-Cas13a system inhibited growth in the HPV16/18 E6-transformed keratinocytes. A significant difference was observed between the OD readings, representing cell growth, of the control group and the CRISPR-Cas13a group in (A) HPV16 E6-transformed keratinocytes and (B) HPV18 E6-transformed keratinocytes. (C) This alteration in cell growth was not observed in keratinocytes transfected with the pcDNA3.1 (+) empty vector. Results are shown as the mean $\pm \mathrm{SD}$. ${ }^{* *} \mathrm{P}<0.01$. Cas, CRISPR-associated protein; HPV, human papillomavirus; OD, optical density.

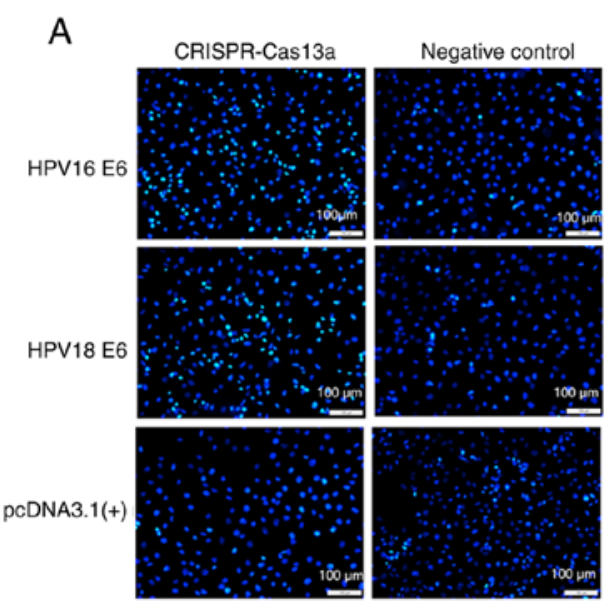

B HPV16 E6-transformed keratinocytes

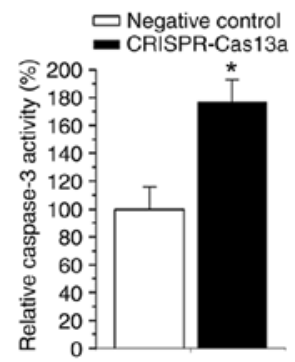

pcDNA3.1(+)-transfected keratinocytes

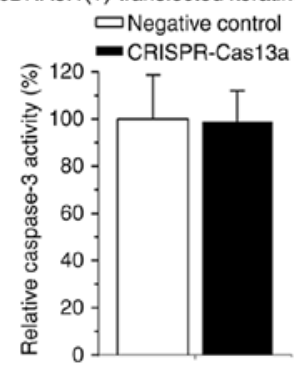

Figure 5. The CRISPR-Cas13a system induced cell apoptosis in the HPV16/18 E6-transformed keratinocytes. Cell apoptosis was measured using (A) Hoechst 33258 staining and (B) ELISAs measuring caspase-3 activity in keratinocytes transfected with CRISPR-Cas13a plasmid targeting HPV16/18 E6 or empty plasmids. Blue stain indicates the nuclei of normal cells whilst green stain indicates nuclei that were undergoing apoptosis. Results are shown as the mean \pm SD. ${ }^{*} \mathrm{P}<0.05$ vs. negative control. Cas, CRISPR-associated protein; HPV, human papillomavirus.

safer gene therapies for HPV-related diseases $(14,15)$. Previous studies have indicated that systemic delivery of CRISPR/Cas9 targeting HPV oncogenes is effective at eliminating established tumors (16-19). However, Cas9 may cause many unexpected off-target effects. The challenge of this technology is to identify Cas nucleases that specifically target functional genes. CRISPR-Cas13a is a newly discovered class of nucleases with a high targeting specificity (6), but to the best of our knowledge it has never been used to target HPV genes.

In the present study, the possibility of using the reprogrammed CRISPR-Cas13a system as a novel molecular agent against the E6 genes of high-risk HPV types (HPV16/18) was investigated. The constructed CRISPR/Cas13a system can break E6 mRNA strands by co-expressing a single Cas13a protein with 
a sgRNA. The present data showed that sgRNAs directed to the mRNA regions could inactivate both HPV-16 E6 and HPV-18 E6. These results highlight the potential for the reprogrammed CRISPR-Cas13a to be used for the treatment of related viruses across various species. From examining the cellular phenotypes, it was found that the reprogrammed CRISPR-Cas13a inhibited the cell proliferation and promoted cell apoptosis in the E6-transformed keratinocytes. These results demonstrated the efficacy of CRISPR-Cas13a in regulating the deregulated phenotypes induced by HPV 16/18. Because the E6 HPV genes share no sequence homology to any human genes, knockdown of this gene will not induce death of healthy cells (16).

Despite the potential application of this research, it is still necessary to continue to test the ability of CRISPR-Cas13a to target HPV genes in animal models. Genetic vectors that deliver Cas13a genes to the target tissues are also yet to be developed.

In conclusion, the present study developed a potent CRISPR-Cas13a system that effectively suppressed cell growth and induced apoptosis in HPV E6-transformed cells, while causing minimal effects in E6-negative human cells. The reprogrammed CRISPR-Cas13a may be further used to inactivate the functions of the HPV E6 gene in future studies.

\section{Acknowledgements}

Not applicable.

\section{Funding}

This study was funded by Foshan Science and Technology Innovation Project (grant no. 1920001000300) and Fujian Provincial Natural Fund (grant no. 2018J01223).

\section{Availability of data and materials}

The datasets used and/or analyzed during the present study are available from the corresponding authors on reasonable request.

\section{Authors' contributions}

JFW and HHC planned and managed the project. CJL, LWG, GQL, MJG, HLW and QQY were involved in performing the experiments and collecting the data. All authors performed a final review of the manuscript and all authors approved the submission of this manuscript.

\section{Ethics and approval}

Informed patient consent was obtained by the parents of the newborn and the patient protocols were approved by the institutional review board of the Foshan Maternal and Child Health Hospital (approval no. 201914258).

\section{Patient consent for publication}

Not applicable.

\section{Competing interests}

The authors declare that they have no competing interests.

\section{References}

1. Remschmidt C, Fesenfeld M, Kaufmann AM and Deleré Y: Sexual behavior and factors associated with young age at first intercourse and HPVvaccine uptake among young women in Germany: Implications for HPV vaccination policies. BMC Public Health 14: 1248, 2014.

2. Ghosh I, Mittal S, Banerjee D, Singh P, Dasgupta S, Chatterjee S, Biswas J, Panda C and Basu P: Study of accuracy of colposcopy in VIA and HPV detection-based cervical cancer screening program. Aust N Z J Obstet Gynaecol 54: 570-575, 2014.

3. Muñoz N, Kjaer SK, Sigurdsson K, Iversen OE, Hernandez-Avila M, Wheeler CM, Perez G, Brown DR, Koutsky LA, Tay EH, et al: Impact of human papillomavirus (HPV)-6/11/16/18 vaccine on all HPV-associated genital diseases in young women. J Natl Cancer Inst 102: 325-339, 2010.

4. McKee CH, Onder Z, Ashok A, Cardoso R and Moroianu J: Characterization of the transport signals that mediate the nucleocytoplasmic traffic of low risk HPV11 E7. Virology 443: 113-122, 2013.

5. Stern PL, van der Burg SH, Hampson IN, Broker TR, Fiander A, Lacey CJ, Kitchener HC and Einstein MH: Therapy of human papillomavirus-related disease. Vaccine 30 (Suppl 5): F71-F82, 2012.

6. Gootenberg JS, Abudayyeh OO, Lee JW, Essletzbichler P, Dy AJ, Joung J, Verdine V, Donghia N, Daringer NM, Freije CA, et al: Nucleic acid detection with CRISPR-Cas13a/C2c2. Science 356: 438-442, 2017

7. Abudayyeh OO, Gootenberg JS, Essletzbichler P, Han S, Joung J, Belanto JJ, Verdine V, Cox DBT, Kellner MJ, Regev A, et al: RNA targeting with CRISPR-Cas13. Nature 550: 280-284, 2017.

8. Zhen S, Hua L, Takahashi Y, Narita S, Liu YH and Li Y: In vitro and in vivo growth suppression of human papillomavirus 16-positive cervical cancer cells by CRISPR/Cas9. Biochem Biophys Res Commun 450: 1422-1426, 2014.

9. Kennedy EM, Kornepati AV, Goldstein M, Bogerd HP, Poling BC, Whisnant AW, Kastan MB and Cullen BR: Inactivation of the human papillomavirus E6 or E7 gene in cervical carcinoma cells by using a bacterial CRISPR/Cas RNA-guided endonuclease. J Virol 88: 11965-11972, 2014.

10. Paner GP, Stadler WM, Hansel DE, Montironi R, Lin DW and Amin MB: Updates in the eighth edition of the tumor-node-metastasis staging classification for urologic cancers. Eur Urol 73: 560-569, 2018

11. Bharti AC, Singh T, Bhat A, Pande D and Jadli M: Therapeutic strategies for human papillomavirus infection and associated cancers. Front Biosci (Elite Ed) 10: 15-73, 2018.

12. Liu DZ, Jin YX, Hou H, Huang YZ, Yang GC and Xu Q: Preparation and identification of activity of anti-HPV-6b/11E1 universal ribozyme-Rz1198 in vitro. Asian J Androl 1: 195-201, 1999.

13. Chen XZ, Zhu KJ, Xu Y, Tang XY, Cai XZ, Zhang X and Cheng H: RNA interference silences the human papillomavirus $6 \mathrm{~b} / 11$ early gene E7 in vitro and in vivo. Clin Exp Dermatol 35: 509-515, 2010.

14. De Buhr H and Lebbink RJ: Harnessing CRISPR to combat human viral infections. Curr Opin Immunol 54: 123-129, 2018.

15. Hsu DS, Kornepati AVR, Glover W, Kennedy EM and Cullen BR: Targeting HPV16 DNA using CRISPR/Cas inhibits anal cancer growth in vivo. Future Virol 13: 475-482, 2018.

16. Zhu D, Shen H, Tan S, Hu Z, Wang L, Yu L, Tian X, Ding W, Ren C, Gao C, et al: Nanoparticles based on poly ( $\beta$-Amino Ester) and HPV16-targeting CRISPR/shRNA as potential drugs for HPV16-related cervical malignancy. Mol Ther 26: 2443-2455, 2018.

17. Yoshiba T, Saga Y, Urabe M, Uchibori R, Matsubara S, Fujiwara $\mathrm{H}$ and Mizukami H: CRISPR/Cas9-mediated cervical cancer treatment targeting human papillomavirus E6. Oncol Lett 17: 2197-2206, 2019.

18. Liu Y, Cai Z and Zhang X: Reprogrammed CRISPR-Cas9 targeting the conserved regions of HPV6/11 E7 genes inhibits proliferation and induces apoptosis in E7-transformed keratinocytes. Asian J Androl 18: 475-479, 2016.

19. Hu Z, Yu L, Zhu D, Ding W, Wang X, Zhang C, Wang L, Jiang X, Shen H, He D, et al: Disruption of HPV16-E7 by CRISPR/Cas system induces apoptosis and growth inhibition in HPV16 positive human cervical cancer cells. Biomed Res Int 2014: 612823, 2014. 